\title{
Strengthening the surveillance of and response to HIV in the Western Pacific
}

\author{
Dongbao Yu and Ying-Ru Lo ${ }^{a}$ \\ Correspondence to Dongbao Yu (e-mail: yud@wpro.who.int).
}

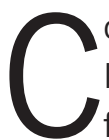
ountries in the Western Pacific Region have lowlevel epidemics - HIV may have been present for many years but never spread to substantial levels in any subpopulation; i.e. prevalence has not been consistently $>5 \%$ in any subpopulation - or concentrated HIV epidemics - the spread of HIV has occurred in a defined subpopulation; i.e. prevalence is consistently $>5 \%$ in a defined subpopulation but is $<1 \%$ in pregnant women in urban areas. ${ }^{1}$ Predominantly affected subpopulations, usually referred to as key populations, in the Region are sex workers (SW), people who inject drugs (PWID), men who have sex with men (MSM) and transgender people (TG). ${ }^{2}$ These populations also show high rates of sexually transmitted infections (STI), which play an important role in enhancing the HIV transmission. ${ }^{3}$ Understanding levels of STI (other than HIV) could help to identify populations for which HIV prevention interventions are needed, especially in areas where HIV prevalence is currently low. $^{2}$ Over the past decades, tremendous efforts and resources have been invested to establish functional HIV surveillance systems in many countries in the Region. ${ }^{4,5}$ However, the same cannot be reported for strengthening STI surveillance. ${ }^{2}$

In this issue, we publish a collection of papers on the surveillance of and response to HIV in the region including assessment of HIV surveillance systems, innovative tools for use in outreach to key populations, interventions for preventing mother-to-child HIV transmission and human resource difficulties for HIV programmes. These papers illustrate the current status and discuss the gaps and challenges for HIV surveillance and programme monitoring in many countries in the Region.

Loo et al. ${ }^{6}$ provides an analysis of HIV surveillance systems of 20 countries in the Asia Pacific region and demonstrates that countries have broadened the number and types of HIV surveillance components over time. This has included introducing population size estimation and integrating behavioural surveys into routine HIV sentinel surveys. Moreover, routine programme monitoring data and case reporting were recently included into some HIV surveillance systems. However, analysis and utilization of surveillance data for programme improvement still lags behind, especially at the subnational level. In a supplemental survey of World Health Organization (WHO) Member States, Yu et al. ${ }^{7}$ report on the coverage and frequencies of HIV serosurveillance surveys among key populations. Female SW are routinely captured by HIV serosurveillance surveys in all seven reported Asian countries; PWID and MSM less so. HIV and STI data among TG are hardly available and often included (if at all) in MSM populations. It is noteworthy that current surveillance systems in most of the Pacific island countries and areas do not include key populations.

Reddy et $a l .^{8}$ discuss the development of a data hub for HIV to promote evidence-informed advocacy and action. It includes a regional HIV database of subnational indicators, a data analysis team and web site with data products and serves as a regional tool to support national and international partners with updated HIV data for policy advocacy and tracking of progress. This large repository contains data from 26 countries in the Asia Pacific region.

The results from these three studies on the improvements of HIV surveillance in the Region are not surprising given the attention and investment from the countries themselves and from the international community over the past two decades. Despite these gains, there are still some areas for strengthening, including the analysis and utilization of surveillance data for programme strengthening, especially at the subnational level. 
One major challenge addressed in this issue is keeping track of outreach efforts to key populations such as PWID, SW and MSM. This has been difficult due to privacy and confidentiality concerns and because these groups can be highly marginalized. Nga et al. ${ }^{9}$ report on an innovative data suite for managing outreach data among key populations in Viet Nam, which includes a unique identifier code, field data collection notebook (the "databook") and a computer data entry system. The databook was found to be inexpensive and can document 40 individual clients, commodity distribution, group contacts and needles/syringe collection. The suite is progressively being used by outbreak workers in more than 40 of the 63 provinces in Viet Nam.

Another important area of HIV prevention is the uptake of interventions for the prevention of mother-tochild transmission (PMTCT) of HIV. Sovannarith et al. ${ }^{10}$ examines the coverage of six key interventions of the PMTCT cascade in 11 operational districts of Cambodia. maternal antiretroviral (ARV) treatment or prophylaxis, delivery in a health facility, infant ARV prophylaxis at birth, infant cotrimoxazole prophylaxis at six weeks, first infant deoxyribonucleic acid-polymerase chain reaction (DNA-PCR) test at six weeks and second infant DNAPCR test at 30 weeks. Programme data from April 2008 to December 2011 revealed critical gaps in monitoring the PMTCT service delivery under the routine programme conditions in Cambodia.

Another article by Rule et al. ${ }^{11}$ highlights that one of the main challenges for the response to HIV in Papua New Guinea is training and maintaining adequate human resources. They identify several workforce issues that need to be addressed.

Although much has been achieved in HIV surveillance and response in the Region, challenges remain. Countries need to make continuous efforts to win the battle against HIV by increasing domestic funding, strengthening surveillance systems and scaling up the coverage of prevention, treatment and care services.

\section{References:}

1. UNAIDS Terminology Guidelines. Geneva, United Nations Joint Programme on HIV/AIDS, 2011 (http://www. unaids.org/en/media/unaids/contentassets/documents/ unaidspublication/2011/JC2118_terminology-guidelines_en.pdf, accessed 20 September 2012).

2. HIV and Sexually Transmitted Infections in the Western Pacific Region 2000-2010. Manila, World Health Organization Regional Office for the Western Pacific, 2012 (http://www.wpro.who. int/publications/2012/document_hiv_and_sti_2000-2010.pdf, accessed 20 September 2012).

3. Fleming DT, Wasserheit JN. From epidemiological synergy to public health policy and practice: the contribution of other sexually transmitted diseases to sexual transmission of HIV infection. Sexually Transmitted Infections, 1999, 75:3-17. doi:10.1136/ sti.75.1.3 pmid:10448335

4. Garcia Calleja JM et al. Has the quality of serosurveillance in low- and middle-income countries improved since the last HIV estimates round in 2007? Status and trends through 2009. Sexually Transmitted Infections, 2010; 86(Suppl 2):ii35-42. doi:10.1136/sti.2010.043653 pmid:21106513

5. Lyerla R, Gouws E, Garcia-Calleja JM. The quality of serosurveillance in low- and middle-income countries: status and trends through 2007. Sexually Transmitted Infections, 2008, 84(Suppl 1):i85-91. doi:10.1136/sti.2008.030593 pmid: 18647872

6. Loo V et al. HIV and AIDS surveillance systems in the Asia Pacific region. Western Pacific Surveillance and Response Journal, 2012, 3(3):9-14. doi:10.5365/wpsar.2012.3.2.007

7. Yu D, Wi T and Garcia Calleja JM. How are countries in the Western Pacific Region tracking the HIV epidemic? Results from a 2011 survey of ministries of health. Western Pacific Surveillance and Response Journal, 2012, 3(3):3-8. doi:10.5365/ wpsar.2012.3.2.010

8. Reddy A, Htin KCW and Shwe YY. HIV and AIDS Data Hub for Asia Pacific: a regional tool to support strategic information needs. Western Pacific Surveillance and Response Journal, 2012, 3(3):18-25. doi:10.5365/wpsar.2012.3.2.005

9. Nga TN et al. Innovative data tools: a suite for managing peer outreach to key affected populations in Viet Nam. Western Pacific Surveillance and Response Journal, 2012, 3(3):29-34. doi:10.5365/ wpsar.2012.3.2.003

10. Sovannarith $\mathrm{S}$ et al. Uptake of interventions for preventing motherto-child HIV transmission in 11 operational districts in Cambodia. Western Pacific Surveillance and Response Journal, 2012, 3(3):22-28. doi:10.5365/wpsar.2012.3.2.009

11. Rule J, Worth H, Roberts $G$ and Taylor R. Human resource challenges in scaling up the response to HIV in Papua New Guinea. Western Pacific Surveillance and Response Journal, 2012, 3(3):15-17. doi:10.5365/wpsar.2012.3.2.006 